\title{
ATM Deficient Tumor
}

National Cancer Institute

\section{Source}

National Cancer Institute. ATM Deficient Tumor. NCI Thesaurus. Code C150447.

A finding indicating that the expression of ATM in a tumor sample is aberrantly low or undetectable. 